\title{
疏水性低共熔溶剂及其在含水体系萃取分离中的 应用
}

熊大珍 ${ }^{1}$, 张倩 $^{1}$, 焚静 $^{2}$, 王键吉 ${ }^{*}$

1. 河南师范大学化学化工学院, 河南省绿色化学重点实验室; 精细化学品绿色制造河南省协同创新中心, 绿色化学介质与反应教育部重点实 验室, 新乡 453007

2. 河南师范大学环境学院, 新乡 453007

*通讯作者, E-mail: Jwang@henannu.edu.cn

收稿日期: 2018-11-16; 接受日期: 2019-01-11; 网络版发表日期: 2019-02-22

国家自然科学基金(编号: 21603063, 21777038)和河南省高等学校重点科研项目(编号: 16A150014)资助

摘要低共熔溶剂是一类新型的绿色溶剂，但目前文献报道的低共熔溶剂大多是亲水性的。它们在水中不稳 定，很难在含水体系中得到应用。为了解决这一问题，近年来疏水性低共熔溶剂受到特别的关注. 本文在介绍低 共熔溶剂基本概念的基础上，以含水体系中短链脂肪胺、低碳醇、双酚A、杀虫剂、重金属和稀有金属的萃取/ 分离为例，综述了近年来疏水性低共熔溶剂在液-液萃取、液相微萃取等分离方面的研究进展，分析了进一步的 发展趋势, 提出了目前尚待解决的主要问题.

关键词绿色溶剂, 低共熔溶剂, 疏水性, 含水体系, 萃取分离

\section{1 引言}

自绿色化学的概念提出以来，科学工作者一直在 积极研究和开发挥发性有机溶剂的绿色替代品. 离子 液体具有蒸汽压低、不可燃、溶解能力强、性质可设 计等优点, 被认为是环境友好的“绿色”介质, 在有机合 成、材料制备、分析分离等领域得到了广泛的应用 ${ }^{[1]}$. 但是，多数离子液体在合成过程中使用了有毒有害的 原料，并有原子利用率低、提纯困难、制备成本高等 问题. 同时，不少离子液体由于生物相容性较差，其 “绿色”性经常遭到质疑 ${ }^{[2]}$. 因此, 开发低毒、低成本的 绿色溶剂成为绿色化学工作者的重要任务之一. 作为
一类新型的绿色溶剂, 低共熔溶剂在某些方面具有比 离子液体更为优越的特性, 在绿色化学和清洁过程中 受到越来越多的关注. 特别是近年来发展的疏水性低 共熔溶剂在可持续分离领域展示出良好的应用前景.

低共熔溶剂(deep eutectic solvents, DESs)是由两 种或两种以上的化合物按照一定的化学计量比通过氢 键作用形成的低共熔混合物, 通常在 $100^{\circ} \mathrm{C}$ 以下的温度 范围内是液体 ${ }^{[2]}$. 这类溶剂是近年来发展起来的一类 新型的绿色溶剂, 许多性质与离子液体类似。但与离 子液体相比，低共熔溶剂制备简单、价格便宜、原子 利用率高、生物相容性好. 从绿色化学的基本原理来 讲，低共熔溶剂更符合绿色溶剂的标准，从而引起科

引用格式: Xiong D, Zhang Q, Fan J, Wang J. Hydrophobic deep eutectic solvents and its application in extraction and separation in aqueous media. Sci Sin Chim, 2019, 49: 933-939, doi: 10.1360/N032018-00251 
学工作者的兴趣和关注. 目前, 低共熔溶剂已在分离过 程、化学反应、功能材料的制备、电化学等领域展示 出良好的应用前景 ${ }^{[1,3]}$. 然而, 文献报道的低共熔溶剂 一般是由亲水化合物组成, 具有较强的水溶性. 由于水 形成氢键的能力较强, 可以不同程度地破坏低共熔溶 剂组分间的氢键 ${ }^{[4,5]}$. 因此, 亲水性低共熔溶剂在水溶 液中不稳定, 不能用于含水体系, 这就极大地限制了低 共熔溶剂的实际应用范围. 因此, 开发疏水性低共熔溶 剂，将低共熔溶剂的应用范围从非水体系扩展至含水 体系显得尤为重要.

2015年，Kroon课题组 ${ }^{[6]}$ 报道了季铵盐-正癸酸类 疏水性的低共熔溶剂, 用于从水溶液中萃取分离短链 的脂肪酸. 几乎与此同时, Marrucho课题组 ${ }^{[7]}$ 报道了 DL-薄荷醇-有机羧酸类疏水性的低共熔溶剂，并用于 从水溶液中萃取分离生物分子. 随后，相关的研究逐 步展开, 但总体上讲, 该领域的研究历史很短, 仍处于 起步阶段. 本文以短链脂肪胺、低碳醇、双酚 $\mathrm{A}$ 、杀 虫剂、重金属和稀有金属的萃取/分离为例，综述了近 年来疏水性低共熔溶剂在含水体系液-液萃取、液相 微萃取等方面的研究进展，分析了疏水性低共熔溶剂 的发展趋势, 提出了目前尚待解决的主要问题.

\section{2 在液-液萃取技术中的应用}

\section{1 短链脂肪酸和低碳醇的萃取}

挥发性脂肪酸指短链的脂肪酸如甲酸、醋酸、丙 酸和丁酸等. 由于挥发性脂肪酸常用于醇类、醛类、 酮类、脂类和烃类等重要化学产品的生产, 因此从水 溶液中回收这些化合物十分重要. 2015年, Kroon课题 组 ${ }^{[6]}$ 率先报道了季铵盐-正癸酸类疏水性低共熔溶剂, 并利用这些疏水性低共熔溶剂从水溶液中分别萃取了 $1 \mathrm{wt} \%$ 的乙酸、丙酸和丁酸. 在这些低共熔溶剂中, 正 癸酸是氢键供体，氢键受体为四丁基氯化铵、四庚基 氯化铵、四辛基氯化铵、四辛基溴化铵、甲基三辛基 氯化铵和甲基三辛基澳化铵等6种季铵盐(图1), 氢键 供体与氢键受体的摩尔比为 $2: 1$. 研究结果表明, 所制 备的疏水性低共熔溶剂对上述三种挥发性脂肪酸的萃 取效率均高于传统的有机溶剂三辛胺, 其中正癸酸-甲 基三辛基氯化铵低共熔溶剂作为萃取剂, 对乙酸、丙 酸和丁酸均具有较高的萃取效率，分别为 $38.0 \%$ 、 $70.5 \%$ 和 $89.8 \%$. 这些低共熔溶剂能从水溶液中萃取解
离的和未解离的羧酸, 扩展了疏水性低共熔溶剂在不 同 $\mathrm{pH}$ 条件下的应用. 此外，随着挥发性脂肪酸烷基链 长度的增加，低共熔溶剂与挥发性脂肪酸的色散作用 增加, 萃取效率也随之增加.

由于能源和环境问题，近年来以乙醇、正丙醇和 正丁醇等低碳醇为代表的生物燃料受到越来越多的关 注. 然而, 由于醇与水存在共沸, 醇-水分离是一个能耗 较高的过程. 2018年, Banerjee等 ${ }^{[8]}$ 使用由薄荷醇和正 十二酸以 $2: 1$ 摩尔比形成的低共熔溶剂分别从水溶液 中萃取 $5 \mathrm{~mol} \%$ $40 \mathrm{~mol} \%$ 的乙醇、 $5 \mathrm{~mol} \% \sim 30 \mathrm{~mol} \%$ 的 正丙醇和 $10 \mathrm{~mol} \%$ $50 \mathrm{~mol} \%$ 的正丁醇. 研究结果表明, 正丁醇的分配系数和选择性均高于乙醇和正丙醇. 此 外, ${ }^{1} \mathrm{H}$ NMR结果显示, 在平衡水相中没有出现低共熔 溶剂, 这表明水相没有被低共熔溶剂污染, 从而有利于 低共熔溶剂的循环使用.

\section{2 双酚 $\mathrm{A}$ 和杀虫剂的萃取}

双酚 $\mathrm{A}$ 是一种雌激素. 水环境中的双酚 $\mathrm{A}$ 是一种持 久性有机污染物, 对人类健康和生态系统安全造成了 潜在的威胁. 2018年, Marrucho课题组 ${ }^{[9]}$ 报道了一类脂 肪酸既作氢键受体又作氢键供体的疏水性低共熔溶 剂, 用于水溶液中双酚 $\mathrm{A}$ 的萃取. 这类低共熔溶剂由不 同比例的正辛酸、正壬酸、正癸酸和月桂酸组成, 具 有黏度低(2 14 mPa s)、密度低等优点, 而且此类疏水 性低共熔溶剂在水中比较稳定. 实验结果显示, 由三个 组分形成的低共熔溶剂的萃取效率均高于由两个组分 形成的低共熔溶剂，一步萃取效率可达到 $79 \%$ \% $1 \%$. 其中由正壬酸、正癸酸和月桂酸以 $3: 1: 1$ 摩尔比形成 的低共熔溶剂的萃取率最高可达到 $91 \%$ 。利用离子液 体双水相体系也可以从水溶液中萃取双酚 $\mathrm{A}$, 而且萃 取率可达到 $100 \%$ ，但是达到相平衡时，富离子液体相 中的离子会进入水相, 从而造成交叉污染. 但是, 羧酸 类疏水性低共熔溶剂在水中的溶解度非常低, 不易引 起水相污染.

人工合成的杀虫剂不仅具有很强的生物毒性，而 且还具有持久性生物累积效应. Marrucho课题组 ${ }^{[10]}$ 以 DL-薄荷醇和四丁基氯化铵为氢键受体，醋酸、乙酰 丙酸、丙酮酸、正丁酸、正己酸、正辛酸、正癸酸和 正十二烷酸等 8 种脂肪酸为氢键供体, 制备了两类疏水 性低共熔溶剂(图2). 在这些低共熔溶剂中, 只有DL-薄 荷醇-辛酸、DL-薄荷醇-癸酸、DL-薄荷醇-正十二烷 


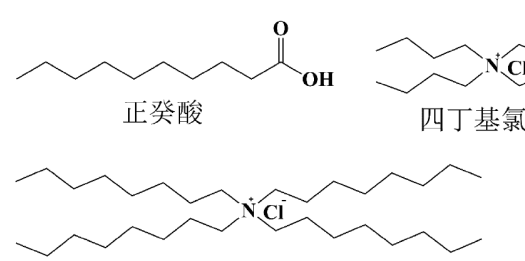

四辛基氯化铵

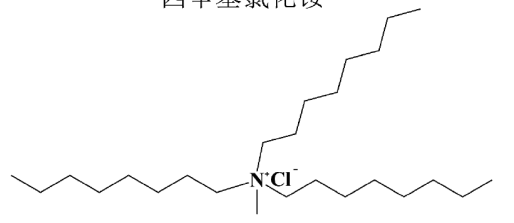

甲基三辛基氯化铵

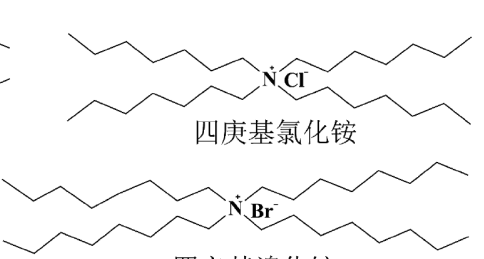

四辛基澳化铵

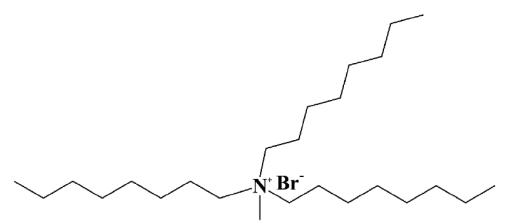

甲基三辛基澳化铵

图 1 季铵盐-正癸酸类疏水性低共熔溶剂组分的结构 ${ }^{[6]}$

Figure 1 Chemical structures of the compounds used for quaternary ammonium-decanoic acid deep eutectic solvents [6].

酸三种低共熔溶剂在水中是稳定的. 利用稳定的疏水 性低共熔溶剂，他们从稀水溶液中萃取了吡虫啉、啶 虫脒、烯啶虫胺和噻虫嗪等4种新烟碱类杀虫剂. 实 验结果表明, 这4种杀虫剂的萃取效率均能达到 $80 \%$, 萃取效率与杀虫剂的疏水性强弱有关. 此外, 低共熔 溶剂循环使用 4 次后, 杀虫剂的萃取效率明显降低.

\section{3 重金属和稀有金属离子的萃取}

利用传统的液-液萃取方法萃取金属离子, 尽管萃 取率很高, 但是萃取过程需要使用大量的有机溶剂, 对 环境造成严重污染. 疏水性低共熔溶剂的出现, 为金属 离子的萃取提供了新的方法. Kroon课题组 ${ }^{[11]}$ 使用疏 水性低共熔溶剂癸酸-利多卡因(摩尔比依次为 $2: 1$ 、 $3: 1$ 和 $4: 1$ )从环境水中萃取钴、铁、锰、镍、锌和铜等 6种过渡金属, 发现这些低共熔溶剂对上述过渡金属的 萃取效率极高, 大多都能达到 $99 \%$ 以上, 且达到萃取平 衡所需时间很短, $5 \mathrm{~s}$ 内就能达到平衡. 可能的萃取机 理为, 在萃取过程中带正电荷的金属离子与部分带正 电荷的利多卡因离子发生离子交换. 为了证实这个推 测, 他们测定了萃取后水相的总有机碳值, 并通过 ${ }^{13} \mathrm{C}$ NMR对萃取后的水相进行分析. 正如预期的一样, 水 相中总有机碳值随着金属盐浓度的增大而增加, 萃取 金属离子后水相中利多卡因的浓度增加. 最后, 向低 共熔溶剂相中加入草酸钠回收低共熔溶剂, 但是只有 摩尔比较高的癸酸-利多卡因低共熔溶剂(3:1和4:1)可 以被回收利用.

金属铟是一种稀有金属, 主要应用于半导体、透 明导电涂层、电子器件、苂光材料、金属有机物等方
面. 铟的分离萃取方法包括萃淋树脂分离法、液膜分 离法、溶剂萃取法等，其中溶剂萃取法在工业上得到 了广泛的应用, 但同样存在环境问题. Tereshatov等 ${ }^{[12]}$ 用4种疏水性低共熔溶剂(四庚基氯化铵-布洛芬、四 庚基氯化铵-油酸、四庚基氯化铵-正癸酸和DL-薄荷 醇-月桂酸, 摩尔比依次为 $7: 3 、 1: 2 、 1: 2$ 和 $2: 1$ ) 作为萃 取剂(图3), 分别从 $0.001 \sim 10.2 \mathrm{~mol} / \mathrm{L}$ 盐酸水溶液和 $1 \times 10^{-7} \sim 8 \times 10^{-1} \mathrm{~mol} / \mathrm{L}$ 草酸水溶液中萃取金属铟. 实验 数据显示, 这些低共熔溶剂对金属铟有良好的萃取性 能, 其中在四庚基氯化铵-布洛芬低共熔溶剂中铟的分 配系数最高可达到 1700 , 而DL-薄荷醇-月桂酸低共熔 溶剂只能从低酸度 $(\mathrm{pH} \approx 3)$ 的水溶中有效萃取铟. 可能 的萃取机理为, 在萃取过程中四烷基铵阳离子或 DL-薄荷醇与金属氯化物形成了离子对. 此外, 向低共 熔溶剂相中加入 $0.1 \mathrm{~mol} / \mathrm{L}$ 二乙烯三胺五乙酸溶液可以 对金属铟进行反萃取, 再生的低共熔溶剂可以循环 使用.

目前虽然疏水性低共熔溶剂已经成功地用于从水 溶液中萃取分离铟和过渡金属, 但是对过渡金属的萃 取没有选择性, 而且再生后的低共熔溶剂未进行下一 步的循环使用. 为了解决对过渡金属的选择性萃取, 最近Coutinho课题组 ${ }^{[13]}$ 使用由便宜的天然资源萜烯和 长链的羧酸形成的疏水性低共熔溶剂在温和的酸性条 件下从其他过渡金属中选择性萃取和分离铜. 这些低 共熔溶剂的氢键受体为薄荷醇和鹿香草酚, 氢键供体 为正辛酸、正癸酸、月桂酸、十四烷酸、十六烷酸和 十八烷酸. 他们研究了羧酸烷基链的长度、氢键供/受 体的摩尔比、水溶液 $\mathrm{pH}$ 、铜的浓度和添加剂盐的种 


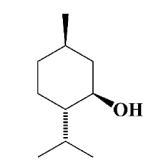

DL-薄荷醇

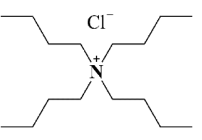

四丁基氯化铵

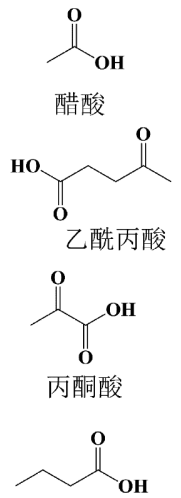

正丁酸

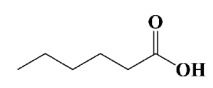

正己酸

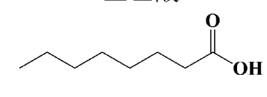

正辛酸

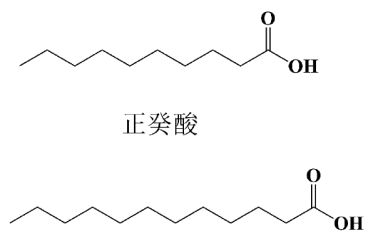

月桂酸
图 $2 \mathrm{DL}$-薄荷醇-脂肪酸和四丁基氯化铵-脂肪酸低共熔溶 剂组分的结构 ${ }^{[10]}$

Figure 2 Chemical structures of the compounds used for DLmenthol-fatty acid and tetrabutylammonium chloride-fatty acid deep eutectic solvents [10].

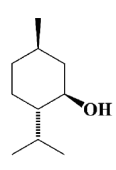

DL-薄荷醇

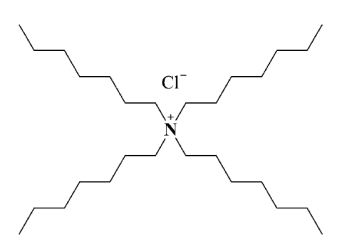

四庚基氯化铵

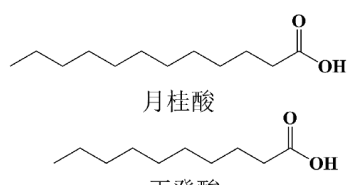

正癸酸

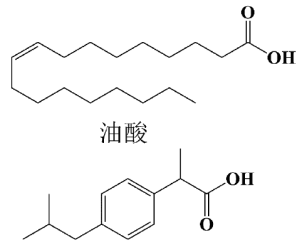

布洛芬
图 3 DL-薄荷醇-有机酸和四庚基氯化铵-有机酸低共熔溶 剂组分的结构 ${ }^{[12]}$

Figure 3 Chemical structures of the compounds used for DLmenthol-organic acid and tetraheptylammonium chloride-organic acid deep eutectic solvents [12].

类对铜萃取率的影响. 实验结果表明, 铜的萃取效率随 着羧酸烷基链长度的增加而降低，随着羧酸摩尔比、 水溶液 $\mathrm{pH}$ 以及铜的浓度的增加而增加．此外，向体系 中加入硫酸钠, 铜的萃取效率增加 $10 \%$, 而加入硝酸钠 和氯化钠对铜的萃取效率无明显影响. 以铜/钴和铜/镍 为例对铜的选择性萃取进行了研究, 发现鹿香草酚-正 癸酸低共熔溶剂对铜的分离具有较高的选择性，在优 化条件下 (正癸酸摩尔分数为 0.7 、水溶液 $\mathrm{pH}$ 值为 4.9、萃取温度为 $20^{\circ} \mathrm{C}$ )，分离因子分别大于 500 和 154 , 远大于阴离子为脂肪酸的季铵盐类离子液体对铜的选 择性. 不过, 该类疏水性低共熔溶剂对铜的选择性萃取
机理目前尚不甚清楚.

\section{3 在液相微萃取技术中的应用}

液相微萃取克服了液-液萃取操作繁琐、使用大 量有机溶剂、对实验人员的健康和环境造成危害等缺 点，是一种集萃取、净化、浓缩于一体的样品前处理 新技术. Zhang等 ${ }^{[14]}$ 将疏水性低共熔溶剂用于饮料中 8 种人工合成色素的液-液微萃取, 在高效液相色谱辅助 下, 定量测定了人工合成色素在水溶液中的含量. 作者 通过实验条件的优化，建立了一个简单、绿色、实用 的饮料中人工色素萃取和测定的新方法. 在优化条件 下， 8 种人工合成色素的检出限为 $0.016 \sim 1.12 \mathrm{ng} / \mathrm{mL}$, 回收率为 $74.5 \% \sim 102.5 \%$, 相对偏差 $<5.4 \%$. 随后, Tang 等 ${ }^{[15]}$ 将由脂肪酸或脂肪醇分别与甲基三辛基氯化铵 形成的两类疏水性低共熔溶剂用于环境水体中左氧氟 沙星和环丙沙星两种抗生素的液-液微萃取, 发现正辛 醇-甲基三辛基氯化铵低共熔溶剂的萃取效果最好, 而 且浴流辅助的方法比加热、超声和微波辅助的方法更 好. 在优化条件下, 左氧氟沙星和环丙沙星的萃取率均 超过 $94.8 \%$.

分散液相微萃取是液相微萃取技术的一种新的萃 取模式，具有操作简单、成本低、回收率高、节约溶 剂和快速等优点. Farajzadeh等 ${ }^{[16]}$ 率先将以氯化胆碱 为氢键受体，4-氯酚、邻甲苯酚、对甲苯酚三种酚类 化合物为氢键供体形成的疏水性低共熔溶剂作为萃取 剂用于果汁和蔬菜中杀虫剂的分散液-液微萃取, 优化 了萃取剂的种类和体积、水相中的离子强度和 $\mathrm{pH}$ 以 及离心速率和时间对杀虫剂萃取的影响. 研究结果表 明，在优化条件下杀虫剂的富集因子范围达到 $312 \sim 482$, 检测限和定量限分别为 $0.46 \sim 3.1 \mathrm{ng} / \mathrm{mL}$ 和

\section{$1.5 \sim 11 \mathrm{ng} / \mathrm{mL}$.}

随后，Zhang 等 ${ }^{[17]}$ 将由甲基三辛基氯化铵和正癸 酸形成的疏水性低共熔溶剂用于水样中紫外防晒剂 (2,4-二羟基二苯甲酮、苯甲酮和2-羟基-4-甲氧基苯甲 酮)的超声辅助分散液-液微萃取, 系统研究了萃取剂 的种类、低共熔溶剂的剂量、超声时间、盐添加剂、 样品体积和离心速率对紫外防晒剂回收率的影响。实 验结果显示, 在萃取过程中不加入分散剂而使用超声 有助于促进精细浊度溶液的形成，明显增加了萃取效 率，缩短了萃取时间. 在优化条件下，2,4-二羟基二苯 
甲酮和苯甲酮在 $0.5 \sim 500 \mathrm{ng} / \mathrm{mL} ， 2$-着基-4-甲氧基苯甲 酮在 $1 \sim 500 \mathrm{ng} / \mathrm{mL}$ 范围内线性良好, 相关系数 $>0.99$, 回 收率为 $90.2 \% \sim 103.5 \%$ ，相对偏差 $<5.9 \%$, 富集因子在 $67 \sim 6$ 之间, 检出限在0.15 0.30 ng/mL. 最后, 将超声 辅助分散液-液微萃取应用于测定游泳池和河水样品 中的紫外防晒剂, 回收率为 $82.1 \% \sim 106.5 \%$.

Boczkaj等 ${ }^{[18]}$ 研究了由天然化合物(DL-薄荷醇、 樟脑、10-十一碳烯酸和正癸酸)制备的疏水性低共熔 溶剂对水溶液中多环芳烃的超声分散液-液微萃取(图 4), 并用气相色谱-质谱法测定了多环芳烃在水样中的 含量. 通过优化重要的萃取参数, 得到的检测限和定量 限分别为 $0.0039 \sim 0.0098 \mu \mathrm{g} / \mathrm{L}$ 和 $0.012 \sim 0.029 \mu \mathrm{g} / \mathrm{L}$, 相对 标准偏差 $<6.09 \%$, 回收率为 $73.5 \% \sim 126.2 \%$. 该方法进 一步用于经过高级氧化技术处理前后的沥青生产的工 业废水, 可定量测定浓度范围在 $0.12 \sim 46.2 \mathrm{~g} / \mathrm{L}$ 的 16 种 多环芳香烃.

最近, Fan课题组 ${ }^{[19]}$ 设计合成了以甲基三辛基氯 化铵为氢键受体、油酸为氢键供体的疏水性低共熔溶 剂, 并将其用作浴旋辅助分散液-液微萃取的萃取剂, 结合高效液相色谱法间接测定了环境水样和生物样品 中的亚硝酸根. 本方法基于在酸性条件下, 亚硝酸根与 对硝基苯胺和二苯胺发生叠氮-偶合反应生成偶氮产 物，通过所合成的疏水性低共熔溶剂浴旋萃取偶氮产 物，间接实现对亚硝酸根的分离、富集和检测. 在优 化条件下, 该方法的线性范围为 $1 \sim 300 \mu \mathrm{g} / \mathrm{L}$, 检出限为 $0.2 \mu \mathrm{g} / \mathrm{L}$, 日内和日间相对标准偏差分别为 $4.0 \%$ 和 $6.0 \%$.

Fan课题组 ${ }^{[20]}$ 还以尼泊金乙酯为供体、甲基三辛 基氯化铵为受体, 合成了一系列疏水性低共熔溶剂, 并 与溶胶-凝胶固相微萃取涂层技术相结合, 制备了一种 疏水性低共溶剂介导的固相微萃取吸附涂层, 考察了 涂层的形貌、热稳定性和选择性，并利用气相色谱评 估了该涂层对挥发性小分子有机污染物甲苯、乙苯和 邻二甲苯的顶空萃取性能. 结果表明, 疏水性低共熔溶 剂可以显著减少溶胶凝胶涂层的开裂和收缩，并在涂 层表面形成均一的多孔形貌. 该涂层对目标分析物具 有良好的富集能力，在优化条件下，方法的线性范围 为 $10 \sim 1000 \mu \mathrm{g} / \mathrm{L}$, 检测限可达 $0.005 \sim 0.025 \mu \mathrm{g} / \mathrm{L}$.

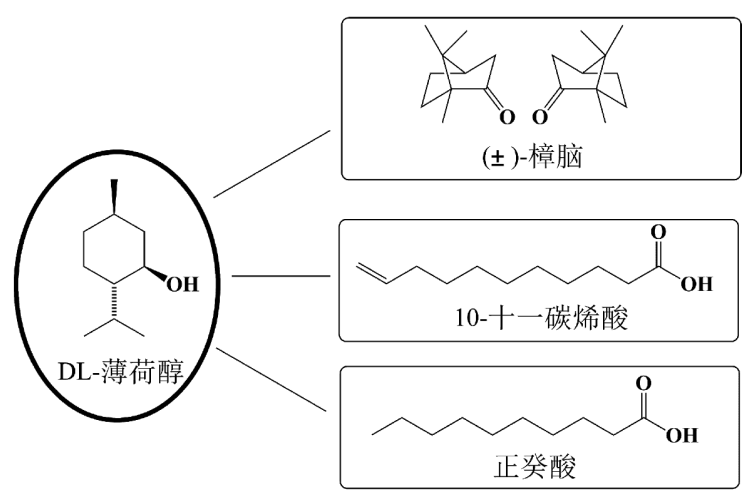

图 4 DL-薄荷醇类低共熔溶剂组分的结构 ${ }^{[18]}$

Figure 4 Chemical structures of the compounds used for DL mentholbased deep eutectic solvents [18].

\section{4 总结与展望}

综上所述, 作为一类环境友好的溶剂, 疏水性低共 熔溶剂在含水体系中短链脂肪酸、低碳醇、双酚A、 杀虫剂、重金属和稀有金属等的液-液萃取/分离方面 展示出良好的应用前景. 不过, 目前仍然有许多重要问 题需要解决. (1) 稳定性问题: 不是所有的疏水性低共 熔溶剂在水中都是稳定的, 只有当氢键供/受体均是疏 水性化合物时, 所制备的疏水性低共熔溶剂在水中才 是稳定的. 如果氢键供体、受体两个组分中有一个是 亲水的, 这个组分就会优先与水作用, 从而破坏低共 熔溶剂. (2) 种类少、萃取的选择性较差: 目前, 疏水 性低共熔溶剂的氢键受体主要集中于有限的季铵盐和 薄荷醇, 氢键供体主要是长链的有机酸, 对于其他类型 的疏水性低共熔溶剂的研究还远远不够. 同时, 萃取分 离的选择性也有待提高. (3) 拓展疏水性低共熔溶剂在 其他领域的应用. 目前, 疏水性低共熔溶剂的应用仅限 于萃取分离技术领域, 应根据对其物理化学性质的研 究结果, 积极扩展在含水体系中的有机合成、材料制 备、电化学等领域的应用. (4) 疏水性低共熔溶剂的 高效再生和循环使用以及相关的萃取机理仍然是需要 研究的问题. 根据结构-性能关系, 利用低共熔溶剂结 构的可调变性, 设计制备功能化的疏水性低共熔溶剂 是下一步的发展方向. 我们相信, 随着研究的不断深 入, 疏水性低共熔溶剂将会有更加广阔的应用前景. 
1 Smith EL, Abbott AP, Ryder KS. Chem Rev, 2014, 114: 11060-11082

2 Zhang Q, De Oliveira Vigier K, Royer S, Jérôme F. Chem Soc Rev, 2012, 41: 7108-7146

3 Mjalli FS, Ahmad O. Thermochim Acta, 2017, 647: 8-14

4 Hammond OS, Bowron DT, Edler KJ. Angew Chem Int Ed, 2017, 56: 9782-9785

5 Dai Y, Witkamp GJ, Verpoorte R, Choi YH. Food Chem, 2015, 187: 14-19

6 van Osch DJGP, Zubeir LF, van den Bruinhorst A, Rocha MAA, Kroon MC. Green Chem, 2015, 17: 4518-4521

7 Ribeiro BD, Florindo C, Iff LC, Coelho MAZ, Marrucho IM. ACS Sustain Chem Eng, 2015, 3: 2469-2477

8 Verma R, Banerjee T. Ind Eng Chem Res, 2018, 57: 3371-3381

9 Florindo C, Romero L, Rintoul I, Branco LC, Marrucho IM. ACS Sustain Chem Eng, 2018, 6: 3888-3895

10 Florindo C, Branco LC, Marrucho IM. Fluid Phase Equilib, 2017, 448: 135-142

11 van Osch DJGP, Parmentier D, Dietz CHJT, van den Bruinhorst A, Tuinier R, Kroon MC. Chem Commun, 2016, 52: 11987-11990

12 Tereshatov EE, Boltoeva MY, Folden CM. Green Chem, 2016, 18: 4616-4622

13 Schaeffer N, Martins MAR, Neves CMSS, Pinho SP, Coutinho JAP. Chem Commun, 2018, 54: 8104-8107

14 Zhu S, Zhou J, Jia H, Zhang H. Food Chem, 2018, 243: 351-356

15 Tang W, Dai Y, Row KH. Anal Bioanal Chem, 2018, 410: 7325-7336

16 Farajzadeh MA, Afshar Mogaddam MR, Aghanassab M. Anal Methods, 2016, 8: 2576-2583

17 Wang H, Hu L, Liu X, Yin S, Lu R, Zhang S, Zhou W, Gao H. J Chromatogr A, 2017, 1516: 1-8

18 Makoś P, Przyjazny A, Boczkaj G. J Chromatogr A, 2018, 1570: 28-37

19 Zhang K, Li S, Liu C, Wang Q, Wang Y, Fan J. J Sep Sci, 2018, 19: doi: 10.1002/jssc.201800921

20 Li T, Song Y, Xu J, Fan J. Talanta, 2019, 195: 298-305 


\title{
Hydrophobic deep eutectic solvents and its application in extraction and separation in aqueous media
}

\author{
Dazhen Xiong $^{1}$, Qian Zhang ${ }^{1}$, Jing Fan $^{2}$, Jianji Wang ${ }^{1 *}$ \\ ${ }^{1}$ Henan Key Laboratory of Green Chemistry, Collaborative Innovation Center of Henan Province for Green Manufacturing of Fine Chemicals, Key \\ Laboratory of Green Chemical Media and Reactions, Ministry of Education, School of Chemistry and Chemical Engineering, Henan Normal \\ University, Xinxiang 453007, China \\ ${ }^{2}$ School of Environment, Henan Normal University, Xinxiang 453007, China \\ *Corresponding author (email: Jwang@henannu.edu.cn)
}

\begin{abstract}
Deep eutectic solvents are a class of new green solvents. However, the deep eutectic solvents reported in literatures so far are mainly limited to hydrophilic deep eutectic solvents, which are not stable in water, and unable to be used in the systems involving water. To resolve this problem, hydrophobic deep eutectic solvents are particularly attractive in recent years. In this work, the concept of deep eutectic solvents is firstly discussed, and then the application of hydrophobic deep eutectic solvents in the extraction/separation of volatile fatty acids, lower alcohols, bisphenol A, pesticides, heavy metals and rare metals from aqueous solutions has been critically reviewed. The developing trend has been analyzed for the hydrophobic deep eutectic solvents based liquid-liquid extraction / separation, and the main issues are suggested for the future researches.
\end{abstract}

Keywords: green solvents, deep eutectic solvents, hydrophobicity, aqueous systems, extraction and separation

doi: $10.1360 / \mathrm{N} 032018-00251$ 